\title{
Techniques for measuring atmospheric aerosols at the High Resolution Fly's Eye experiment
}

\author{
R. Abbasi, ${ }^{\text {a }}$ T. Abu-Zayyad, ${ }^{\text {a }}$ J.F. Amann, ${ }^{\text {e }}$ G.C. Archbold, ${ }^{\text {a }}$ \\ K. Belov, ${ }^{\text {a }}$ S. BenZvi, ${ }^{\text {d }}$ J.W. Belz,${ }^{\text {f }}$ D.R. Bergman, ${ }^{\mathrm{g}}$ J. Boyer, ${ }^{\mathrm{c}}$ \\ C.T. Cannon, ${ }^{\mathrm{a}}$ Z. Cao, ${ }^{\mathrm{a}}$ B.M. Connolly, ${ }^{\mathrm{d}}$ J. Fedorova, ${ }^{\mathrm{a}}$ \\ C.B. Finley, ${ }^{\mathrm{d}}$ J.H.V. Girard, ${ }^{\mathrm{a}}$ R.C. Gray, ${ }^{\mathrm{a}}$ W.P. Hanlon, ${ }^{\mathrm{g}}$ \\ C.M. Hoffman, ${ }^{\text {e }}$ M.H. Holzscheiter, ${ }^{\mathrm{g}}$ G.A. Hughes, ${ }^{\mathrm{e}}$ \\ P. Hüntemeyer, ${ }^{a}$ C.C.H. Jui, ${ }^{a}$ K. Kim, ${ }^{a}$ M.A. Kirn,,${ }^{f}$

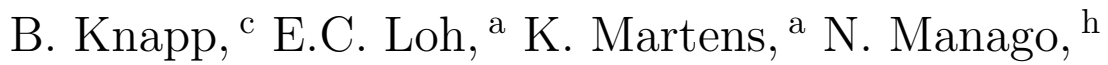 \\ E.J. Mannel, ${ }^{\text {c }}$ J.A.J. Matthews, ${ }^{\text {b }}$ J.N. Matthews, ${ }^{\text {a }}$ \\ J.R. Mumford, ${ }^{\mathrm{a}}$ A. O'Neill, ${ }^{\mathrm{d}} \mathrm{R}$. Riehle, ${ }^{\mathrm{a}} \mathrm{K}$. Reil, ${ }^{\mathrm{a}}$ \\ M.D. Roberts*, ${ }^{\text {b M. Seman, }}{ }^{\text {d S.R. Schnetzer, }}{ }^{\mathrm{g}}$ P. Shen, ${ }^{\mathrm{a}}$ \\ G. Sinnis ${ }^{e}$ J.D. Smith, ${ }^{\text {a }}$ P. Sokolsky, ${ }^{\text {a }}$ C. Song, ${ }^{\mathrm{d}}$
} R.W. Springer, ${ }^{a}$ B.T. Stokes, ${ }^{a}$ S.B. Thomas, ${ }^{a}$ G.B. Thomson, ${ }^{g}$ D. Tupa, ${ }^{\text {e }}$ S. Westerhoff, ${ }^{\text {d }}$ L.R. Wiencke**, ${ }^{\text {a }}$ A. Zech ${ }^{\mathrm{g}}$

${ }^{a}$ High Energy Astroparticle Physics Institute, University of Utah, Salt Lake City, $U T$

${ }^{\mathrm{b}}$ Department of Physics, University of New Mexico, Albuquerque, NM

${ }^{\mathrm{c}}$ Columbia University, Nevis Labs, Irvington, $N Y$

d Department of Physics, Columbia University, New York, NY

${ }^{\mathrm{e}}$ Los Alamos National Laboratory, Los Alamos, NM

${ }^{\mathrm{f}}$ Department of Physics, Montana State University,Bozeman, MT

${ }^{g}$ Department of Physics and Astronomy, Rutgers University, Piscataway, NJ

${ }^{\mathrm{h}}$ University of Tokyo, Institute for Cosmic Ray Research, Kashiwa, Japan

*Corresponding author email: roberts@cosmic.utah.edu

**Corresponding author email: wiencke@cosmic.utah.edu

Abstract

We describe several techniques developed by the High Resolution Fly's Eye experiment for measuring aerosol vertical optical depth, aerosol horizontal attenuation 
length, and aerosol phase function. The techniques are based on measurements of side-scattered light generated by a steerable ultraviolet laser and collected by an optical detector designed to measure fluorescence light from cosmic-ray air showers. We also present a technique to cross-check the aerosol optical depth measurement using air showers observed in stereo. These methods can be used by future air fluorescence experiments.

Key words: air showers; atmospherics; aerosols; lasers; uv; LIDAR

\section{Overview}

Cosmic-ray air fluorescence experiments measure light produced by high-energy air showers. The technique is calorimetric, with the atmosphere as the calorimeter. The integrated scintillation light produced by an extensive air shower is proportional to the the primary particle energy and is almost independent of the primary particle composition. However, once this light is produced, the amount that reaches the observatory depends on how this light propagates from the shower through the molecular and aerosol components of the atmosphere to the light-collection optics of the detector. The benefit of the atmosphere that makes the air fluorescence technique possible is balanced by the challenge to understand how the light propagates through the atmosphere.

Several aspects of the High Resolution Fly's Eye (HiRes) experiment address this challenge. To reduce atmospheric effects, the experiment is located in a remote desert where the average humidity and cloud cover are relatively low. The detector stations are located on hills approximately $100 \mathrm{~m}$ above the desert floor. This places the optical detectors above many aerosols, including low-lying dust and ground fog. To measure atmospheric effects, the experiment includes steerable uv lasers that probe the aperture of the detector while the experiment is running. The light scattered out of a laser beam produces tracks in the same detectors that measure tracks of uv light from air showers. Changes in atmospheric aerosols change the amount of light scattered and the amount of light that reaches the detector. Modeling the detector, the laser, and the atmosphere generates a simulated set of detector measurements. The simulated data are compared and fit to the actual detector measurements of the laser. This procedure yields a set of parameters that describe the aerosols. 


\section{Components of the Atmosphere}

Most of the light observed from air showers is produced in the the troposphere. With regard to atmospheric fluorescence measurements, the atmosphere has two main components: the molecular atmosphere and aerosols. Both components can be described in terms of optical depths, denoted $\tau_{M}$ and $\tau_{A}$. Assuming single scattering, the transmission through a vertical column of atmosphere can be expressed as $T=\exp \left(-\tau_{M}-\tau_{A}\right)$.

\subsection{The Molecular Component}

The molecular atmosphere, comprising mainly nitrogen and oxygen molecules, has absorption and scattering properties that are well understood. Because the 300 to $400 \mathrm{~nm}$ wavelengths of interest (see Kakimoto et al. (1996) and Nagano et al. (2004)) are much larger than the nitrogen and oxygen molecules, the electric field can be approximated as constant across a molecule, and consequently, light propagation can be described using Rayleigh scattering theory [see, for example, Naus and Ubachs (2000), Miles et al. (2001), and Bucholtz (1995)]. The molecular component is relatively stable, with small variations due to height-dependent changes in temperature and pressure. Because a purely molecular atmosphere has the the clearest and best-understood viewing conditions, it provides a boundary value solution to light-propagation studies. Air density can be obtained by applying the ideal gas law to radiosonde measurements (collected at the Salt Lake, UT, and Reno, NV, airports) of pressure and temperature as a function of height. The dependence on height follows an adiabatic model, with variations at the $5 \%$ level caused by temperature and pressure changes associated with different seasons and weather systems. For circularily or unpolarized light, the scattering probability as a function of scattering angle, $\theta$, follows $1+\cos ^{2}(\theta)$, with higher-order corrections that are negligible for the purposes of this work.

Applying the methods described in Bodhaine et al. (1999) to Salt Lake radiosonde data, yield values for $\tau_{M}$ at $355 \mathrm{~nm}$, a wavelength that falls near the middle of the air fluorescence spectrum. $\tau_{M}$ from the $1.5 \mathrm{~km}$ elevation of HiRes $\left(850 \mathrm{~g} / \mathrm{cm}^{2}\right)$ through the entire atmosphere is 0.50 . This value drops to 0.37 through $10 \mathrm{~km}\left(223 \mathrm{~g} / \mathrm{cm}^{2}\right)$ above ground, and to 0.17 through $3.6 \mathrm{~km}(557$ $\mathrm{g} / \mathrm{cm}^{2}$ ) above ground, with seasonal fluctuations of 0.005 . For comparison, we note the average $\tau_{A}$ between ground and a $3.5 \mathrm{~km}$ height was measured at $355 \mathrm{~nm}$ to be 0.04 (see section 4 ). 


\subsection{The Aerosol Component}

The aerosol component is more complicated. Aerosol sizes range from $10^{-6}$ $\mathrm{cm}$ (large molecules) to $10^{-3} \mathrm{~cm}$ (particulate matter). The distribution of aerosols changes with location, height, and time. Local dust can be lifted from the desert floor by wind and transported to elevations typically less than 1 $\mathrm{km}$. More distant sources can create aerosols that may be carried over large areas by upper-level air flows. A precise analytic description of absorption and scattering is impossible to obtain because of the variable nature of the aerosol size distributions and dielectric constants. Light scattering from aerosols of a specific size and spherical shape can be described by Mie scattering theory (Bohren and Huffman (1983)). In general, the scattering probability as a function of angle is characterized by a forward peak, relatively little scattering at 90 degrees, and some enhancement for backward scattering. However, because the precise details of aerosol size, shape, and distribution are not known a priori, one must rely on phenomenologically based models for guidance. A commonly used simulation package, MODTRAN (Moderate Resolution Transmission), was developed by the US Air Force (Longtin (1988)). The package contains aerosol models for different regions including a "US Standard Desert" parameterization.

In contrast to the molecular atmosphere, aerosols are known to vary on short time scales. Weather fronts can load the atmosphere with wind-driven aerosols. Rain and snowstorms can flush haze and dust from the atmosphere, often leaving near-molecular viewing conditions. The aerosol density at ground level, the vertical distribution of the aerosols, and the scattering and absorption properties can all change within one night of observing. Atmospheric monitoring at HiRes therefore entails measuring the time dependence of the distribution of aerosols in the detector aperture and determining their gross properties to the level required to reconstruct air showers.

In this article, we describe the measurement of three aerosol quantities: vertical aerosol optical depth $\left(\tau_{A}\right)$, horizontal aerosol scattering length at detector level (HAL), and aerosol phase function. $\tau_{A}$, measured from the elevation of the detector to an elevation of $3.5 \mathrm{~km}$, is the most important quantity of the three for several reasons. First, because the field of view of a HiRes detector begins at an elevation angle of 3 degrees, and because the HiRes stations are on hills, none of the aperture extends to ground level. At a distance of 19 $\mathrm{km}$, the lower edge of the aperture is $1 \mathrm{~km}$ above detector station height. Second, $\tau_{A}$ describes the total aerosol loading, and most of the aerosols lies below the height at which most of the light is observed from air showers, especially for showers above $10 \mathrm{EeV}$, which tend to be further away. Therefore, under the assumption of horizontal aerosol uniformity, (also addressed in this article), most of the light reaching the detector is sensitive to the total amount 
of aerosols; the correction for aerosol transmission depends more on elevation angle than on distance. As viewed along the optical axis of a lowest ring HiRes detector, an uncertainty of 0.02 in $\left(\tau_{A}\right)$ corresponds to an uncertainty of $11 \%$ in transmission. Over the full 30-3 degree range of elevation angles, this uncertainty changes from $4 \%$ at the very top to $32 \%$ at the very bottom. For air-showers, these uncertainties translate to an uncertainty in energy reconstruction of order 10 to $20 \%$.

\section{Apparatus}

Measurements of $\tau_{A}$ and HAL are determined from laser tracks recorded by the HiRes detectors.

\subsection{The Steerable Laser System}

The steerable laser system, dubbed HR2SLS Wiencke et al. (1999), fires a pattern of 1000-1300 shots per hour. Vertical shots can be used to extract vertical aerosol optical depth. Horizontal attenuation lengths can be extracted from near-horizontal shots fired across the HiRes1 detector station. A pattern of elevated shots fired in 15-degree steps in azimuth may be used to study atmospheric horizontal uniformity.

The laser produces a $7 \mathrm{~ns}$ wide pulse of light at $355 \mathrm{~nm}$. This wavelength closely matches the $357 \mathrm{~nm}$ fluorescence line. The maximum beam energy is $7 \mathrm{~mJ}$. The beam can be steered in any direction above the horizon. The direction, energy, and time of each pulse are measured and recorded locally. The laser firing times are synchronized using a GPS clock so that laser events can be isolated from other HiRes triggers in a physics-blind manner. Different energies and polarizations can be selected using filter wheels that provide a combination of quarter wave-plates and attenuation filters.

One percent of the beam is sampled by a photo diode detector to obtain a relative measurement of the pulse by pulse energy. Special measurements are taken at regular intervals to determine the absolute calibration. For these measurements, an energy-absorbing pyroelectric probe is placed temporarily where the beam exits to the sky. The pyroelectric probe is periodically recalibrated to $5 \%$ by the manufacturer using an NIST traceable system.

Circularly polarized beams with energies from 0.05 to $3 \mathrm{~mJ}$ were used in obtaining measurements. Circular polarization was chosen over linear polarization to reduce polarization dependent scattering effects and simplify simu- 
lations. The energy range must match the detector dynamic range for different beam directions. For example, vertical shots needed to measure $\tau_{A}$ are viewed at a distance of $12.7 \mathrm{~km}$. These shots require energy of 1-3 mJ. On the other hand, the horizontal shots needed to measure HAL must pass within a few hundred meters of the detector. These shots require a much lower energy, typically $0.1 \mathrm{~mJ}$, to avoid detector saturation. For each combination of filter and direction setting, the laser fires 15 shots which are later averaged to reduce statistical uncertainties.

\subsection{The HiRes Detectors}

The detectors that view the laser tracks are described briefly. HiRes consists of two stations, HiRes1 and HiRes2, which are separated by $12.6 \mathrm{~km}$. The analysis described here used detectors (Abu-Zayyad et al. 2000) at the HiRes1 station. These detectors are arranged to view nearly 360 degrees in azimuth from an elevation angle of 3-17 degrees. A spherical mirror (3.78 $\mathrm{m}^{2}$ effective area) at each detector reflects light from the night sky through a uv pass filter onto a cluster of 256 hexagonal photomultiplier tubes (PMTs). Each PMT views about $1 \times 1^{\circ}$ of night sky. As a laser light pulse passes upward through the field of view of a mirror, light scattered from the beam is collected and focused to a spot that crosses downward across the cluster, triggering PMTs along its path. The nominal spot is about the size of a PMT. The nominal PMT gain is $10^{5}$, and the nominal quantum efficiency is $25 \%$. The signal in a PMT from laser shots (and air showers) is typically hundreds to thousands of photoelectrons.

The detector readout system at HiRes1 uses sample-and-hold electronics. Signal digitization and data formatting are performed by local electronics at each mirror. PMT outputs are AC coupled. The signal-processing channel used for this work has a three-pole Bessel filter with a $375 \mathrm{~ns}$ time constant and a charge integration gate of $5.6 \mu \mathrm{s}$. TDCs measure times between PMT triggers by integrating a gated constant-current source. Discriminator thresholds are adjusted every 4 seconds to maintain a count rate of $200 \mathrm{~Hz}$. The trigger requirement for a signal digitization is a sixfold coincidence between PMTs. Typical trigger rates are $5-10 \mathrm{~Hz}$ per mirror. System dead-time is less than $2 \%$.

\section{Determining Vertical Aerosol Optical Depth}

Under the assumptions of horizontal uniformity, and single scattering, the transmission, $T_{A}$, through an optical depth of aerosols, $\tau_{A}$, at an elevation 


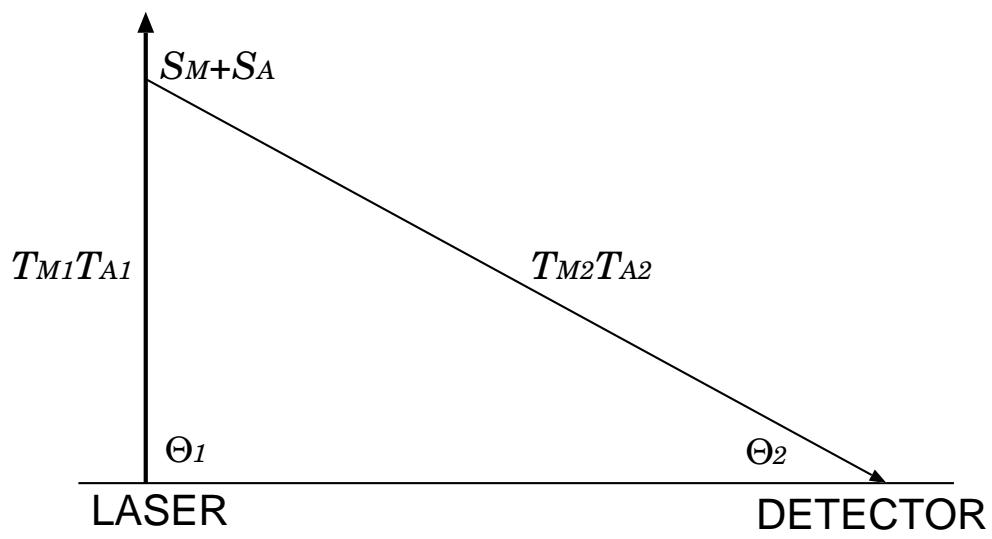

Fig. 1. Geometry for measuring aerosol optical depth.

angle $\theta$ is

$$
T_{A}=\exp \left(-\frac{\tau_{A}}{\sin (\theta)}\right)
$$

This equation includes both absorption and scattering. For the case that all the aerosols are between the starting and ending elevation of interest, and the aerosols are uniform horizontally, $T_{A}$ is independent of the aerosol vertical distribution.

The method to determine $\tau_{A}$ normalizes the measurement of a laser beam to the measurement that would be expected under aerosol-free (i.e. molecular) atmospheric conditions. Figure 1 illustrates the geometrical configuration of the laser and the detector.

The amount of light from the laser that reaches the detector can be written as

$$
N_{O B S}=N_{\gamma L} T_{M 1} T_{A 1}\left(S_{A}+S_{M}\right) T_{M 2} T_{A 2}
$$

where $N_{O B S}$ is the number of photons observed, $N_{\gamma L}$ is the number of photons emitted per laser pulse, $T_{M 1}$ and $T_{A 1}$ are the molecular and aerosol transmission components from the laser to the point of scatter, $S_{A}$ is the fraction of photons scattered toward the detector by aerosols, $S_{M}$ is the fraction scattered by the molecular component, and $T_{M 2}$ and $T_{A 2}$ are the molecular and aerosol transmission components from the point of scatter to the detector. Normalized to the prediction for a purely molecular atmosphere $\left(N_{M O L}=N_{\gamma_{L}} T_{M 1} S_{M} T_{M 2}\right)$, equation 2 becomes

$$
\frac{N_{O B S}}{N_{M O L}}=T_{A 1} T_{A 2}\left(1+\frac{S_{A}}{S_{M}}\right)
$$


Applying equation 1 yields

$$
\tau_{A}=\frac{-1}{\frac{1}{\sin \theta_{1}}+\frac{1}{\sin \theta_{2}}}\left(\ln \left(\frac{N_{O B S}}{N_{M O L}}\right)-\ln \left(1+\frac{S_{A}}{S_{M}}\right)\right)
$$

The two unknowns, $\tau_{A}$ and $S_{A}$, illustrate the ambiguity between scattering and transmission inherent in conventional LIDAR measurements. The ambiguity can be eliminated if the scattering occurs in a region where $S_{A}$ is small compared to $S_{M}$. Equation 4 then simplifies to

$$
\tau_{A}=-\frac{\sin \theta_{1} \sin \theta_{2}}{\sin \theta_{1}+\sin \theta_{2}} \ln \left(\frac{N_{O B S}}{N_{M O L}}\right)
$$

In this case, $\tau_{A}$ becomes a simple function of geometry and the number of observed photons that are normalized to $N_{M O L} . N_{M O L}$ can be determined either by Monte Carlo simulation, or from data collected under molecular conditions. The latter method does not require an absolute calibration of either the laser or the detector. However, if the reference data were collected during a period when aerosols were present, the measured value of $\tau_{A}$ will be lower than the actual value. Normalizing to simulated data requires absolute calibration of the laser and detector and simulation of light propagation through a molecular atmosphere. This analysis was performed with data normalization and with Monte Carlo normalization. The results are shown in figures 2 and 3.

The calculation of $N_{O B S}$ averages over sets of 15 vertical laser shots fired each hour at two energy settings. A region of PMTs was selected at the top of the track. For the data-normalized analysis, the region was 2 PMTs wide by 4 PMTs high, corresponding to an elevation of $3.5 \mathrm{~km}$ above the laser. For the Monte Carlo-normalized analysis, a 2x2 PMT grid was used, with a corresponding elevation of $3.8 \mathrm{~km}$.

$N_{M O L}$ for the Monte Carlo normalized analysis included effects of multiplescattering, estimated to be at the $5 \%$ level for a molecular atmosphere. To reduce computation time, the Monte Carlo simulation exploited the symmetry of a vertical laser beam. $N_{M O L}$ for the data normalized analysis used data collected on December 12, 2000, UT hour 6.

The data sample in this analysis extends from September of 1999 to May 2001. Figure 2 shows the time evolution of hourly $\tau_{A}$ measurements obtained by the two methods of analysis, and the difference between the measurements. The projection of these data onto the $\tau_{A}$ axis and the correlation between them are shown in figure 3 . The systematic difference of 0.009 between the two analyses could be caused by the presence of aerosols during the reference "clear period" or by the relative calibration between the laser and the detector. For reference, 0.009 corresponds to a calibration uncertainty of approximately $5 \%$. 

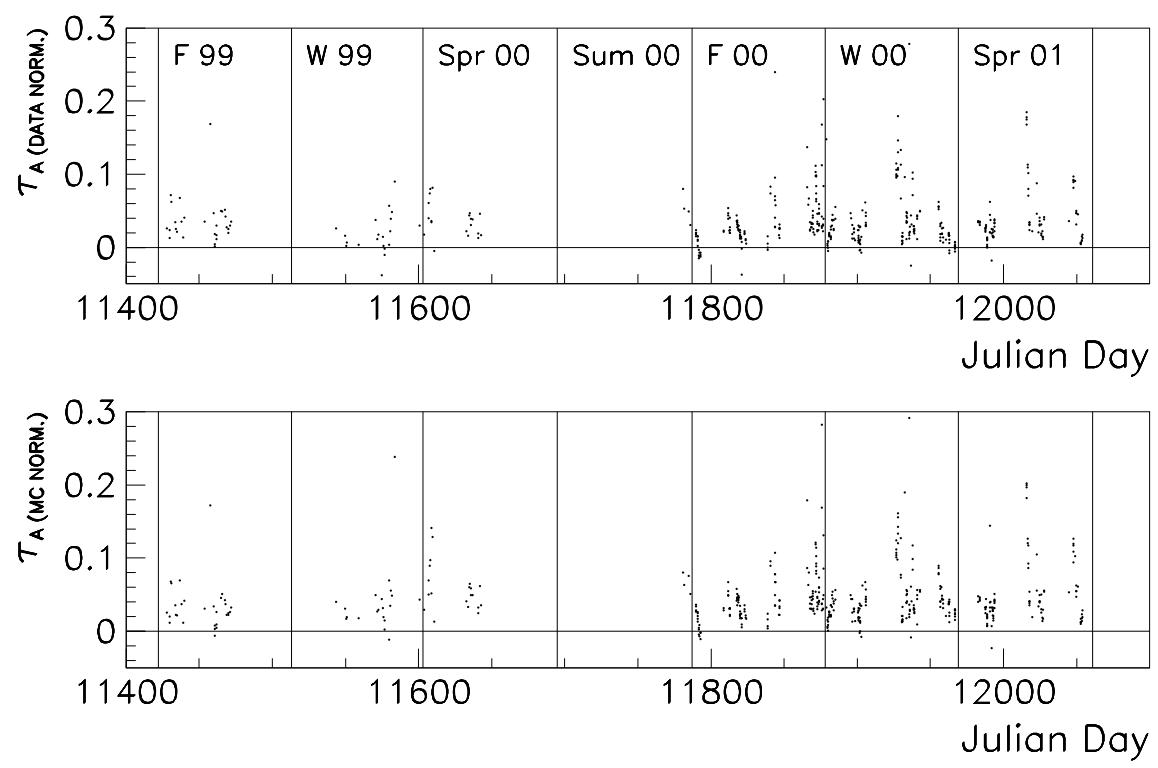

(B)

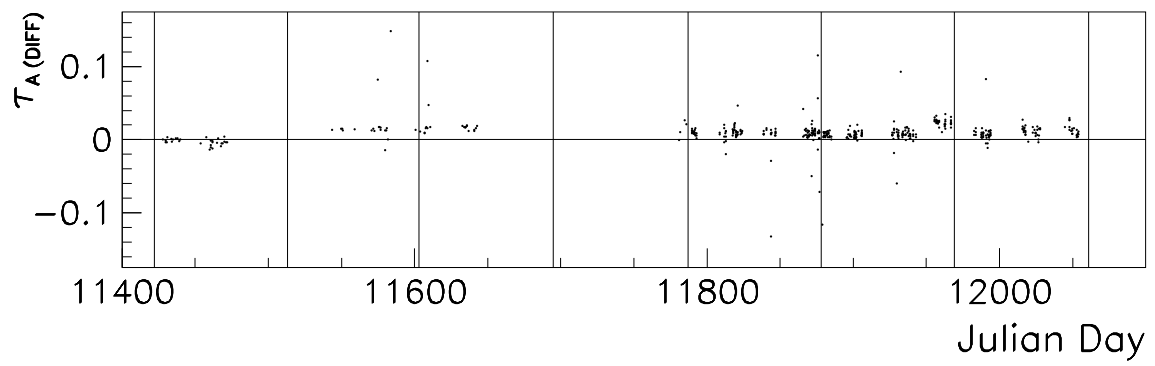

(C)

Fig. 2. Time evolution of $\tau_{A}$. The top panel (A) shows the data-normalized measurements. The middle panel (B) shows the Monte Carlo-normalized measurements, and the bottom panel $(\mathrm{C})$ shows the difference.

The asymmetric shape of the $\tau_{A}$ distributions in figure 3 are consistent with a molecular limit (at $\tau_{A}=0$ ), and a tail on the high side due to aerosol loading. The means of the $\tau_{A}$ distributions are 0.036 and 0.046 . Their full widths at half maxima are 0.035 .

We have identified three sources of systematic error that dominate the measurement uncertainty. A $10 \%$ uncertainty in the relative calibration between the laser and the detector contributes a systematic error of 0.02 to the Monte Carlo-normalized $\tau_{A}$ average. A $5 \%$ time dependent variation in the gains of the PMTs used in the analysis contribute 0.01 systematic error to both measurements. Finally, the reference aerosol-free normalization data contributes an uncertainty of $0.01 \tau_{A}$ to the data-normalized measurement. The number of sets of laser shots analyzed reduces the statistical error of the mean to 0.001. Adding the systematic errors in quadrature yields 

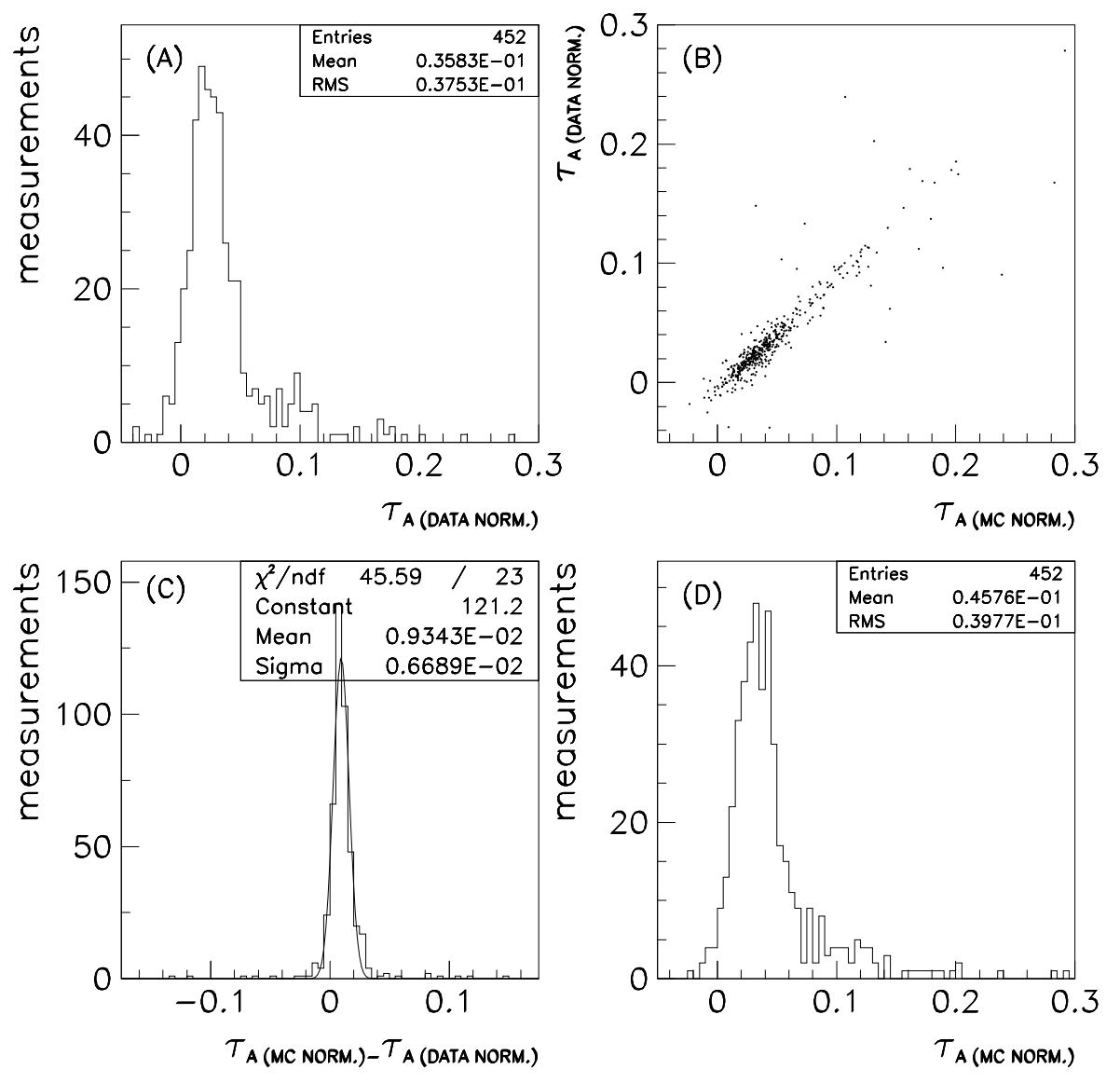

Fig. 3. Vertical aerosol optical depth measured at $355 \mathrm{~nm}$. The upper left (A) and lower right (D) panels show the measurement using the data-normalized and the Monte Carlo-normalized techniques. Panels (B) and (C) show the correlation and the difference between the measurements.

Mean $\tau_{A}=0.046 \pm 0.022_{\text {sys }} \pm 0.001_{\text {stat }}$ (Monte Carlo-normalized)

Mean $\tau_{A}=0.036 \pm 0.014_{\text {sys }} \pm 0.001_{\text {stat }}$ (data-normalized)

We note that equation 1 yields a correction factor of 1.25 for light traveling along the optical axis of a HiRes1 detector through a $\tau_{A}$ of 0.04 .

To study the vertical distribution of aerosols, the analysis was repeated using sets of PMTs corresponding to different elevations. The mean difference between $\tau_{A}$ for $0-3.5 \mathrm{~km}$ and $0-2.5 \mathrm{~km}$ was found to be less than 0.0025 . See figure 4. The mean $\tau_{A}$ for $0-1.5 \mathrm{~km}$ was 0.008 smaller than mean $\tau_{A}$ for $0-3.5 \mathrm{~km}$. These numbers indicate that the bulk of the aerosols during this period were distributed in the lower $1.5 \mathrm{~km}$ of the atmosphere. They are also consistent 

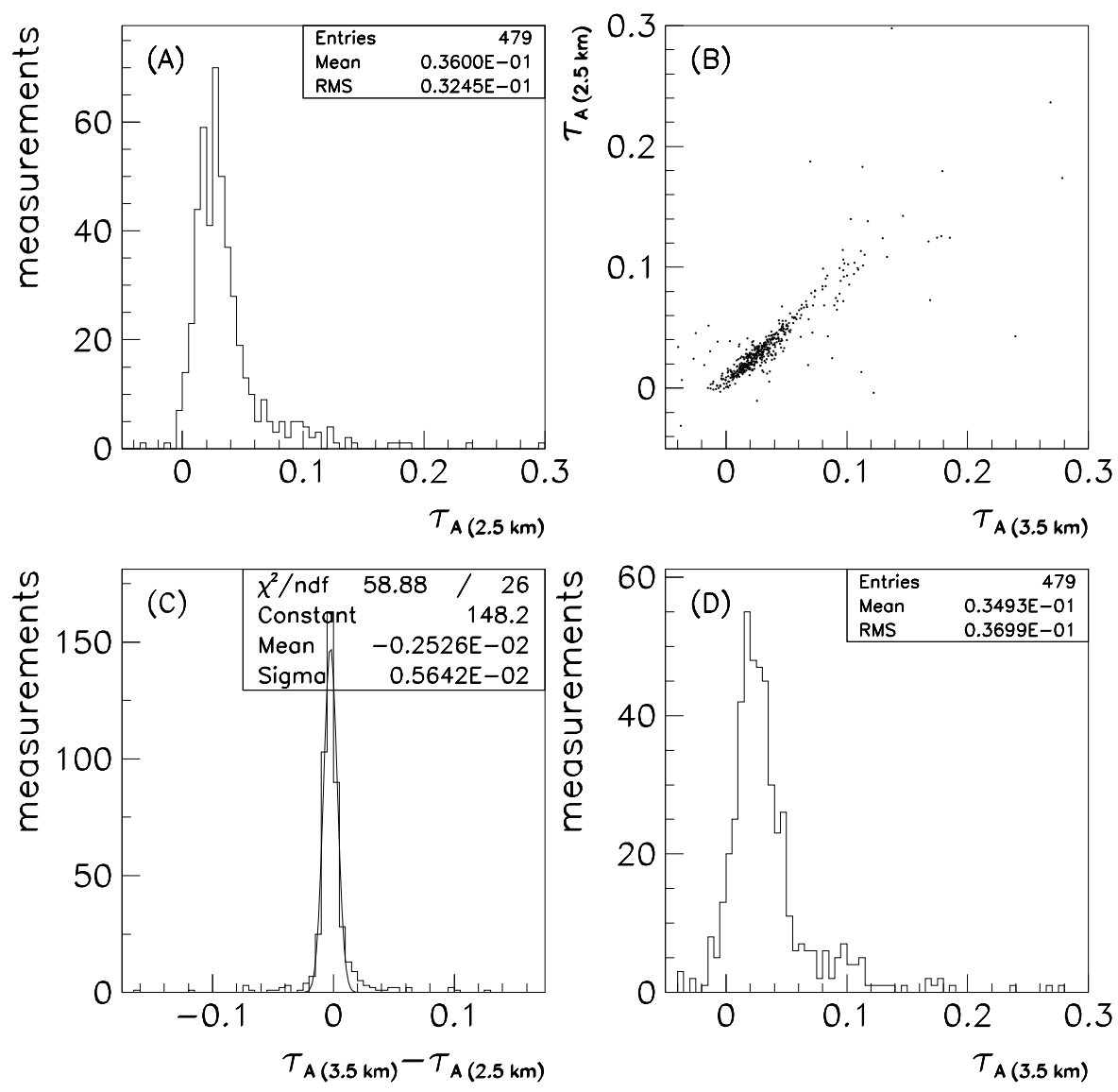

Fig. 4. Correlation (panel B) between aerosol optical depth as measured from $\mathrm{z}=$ 0 to $2.5 \mathrm{~km}$ (panel A) and from $\mathrm{z}=0$ to $3.5 \mathrm{~km}$ (panel D). This analysis uses data normalization. If the average $0.04 \tau_{A}$ was uniformly distributed in height, an average difference of approximately 0.01 would have been observed. This "uniform height" model is ruled out because the difference (panel $\mathrm{C}$ ) is much smaller.

with an average aerosol scale height of approximately $1 \mathrm{~km}$.

The optical-depth technique can also be applied to inclined laser tracks. To test horizontal uniformity, we compared optical-depth measurements from two laser directions that were symmetric about the line between the laser and the HiRes1 detector station. The pattern of laser shots includes shots of 15 degrees elevation angle and azimuth angles of \pm 75 degrees with respect to HiRes1. The portions of the tracks used in this measurement are $3 \mathrm{~km}$ above the ground,and are separated by roughly $20 \mathrm{~km}$. Although the separation is less than $20 \mathrm{~km}$ over most of the symmetric light paths (the light starts at the laser and ends at the HiRes1 detector), the symmetric measurements still probe different parts of the HiRes aperture and are also made by different 

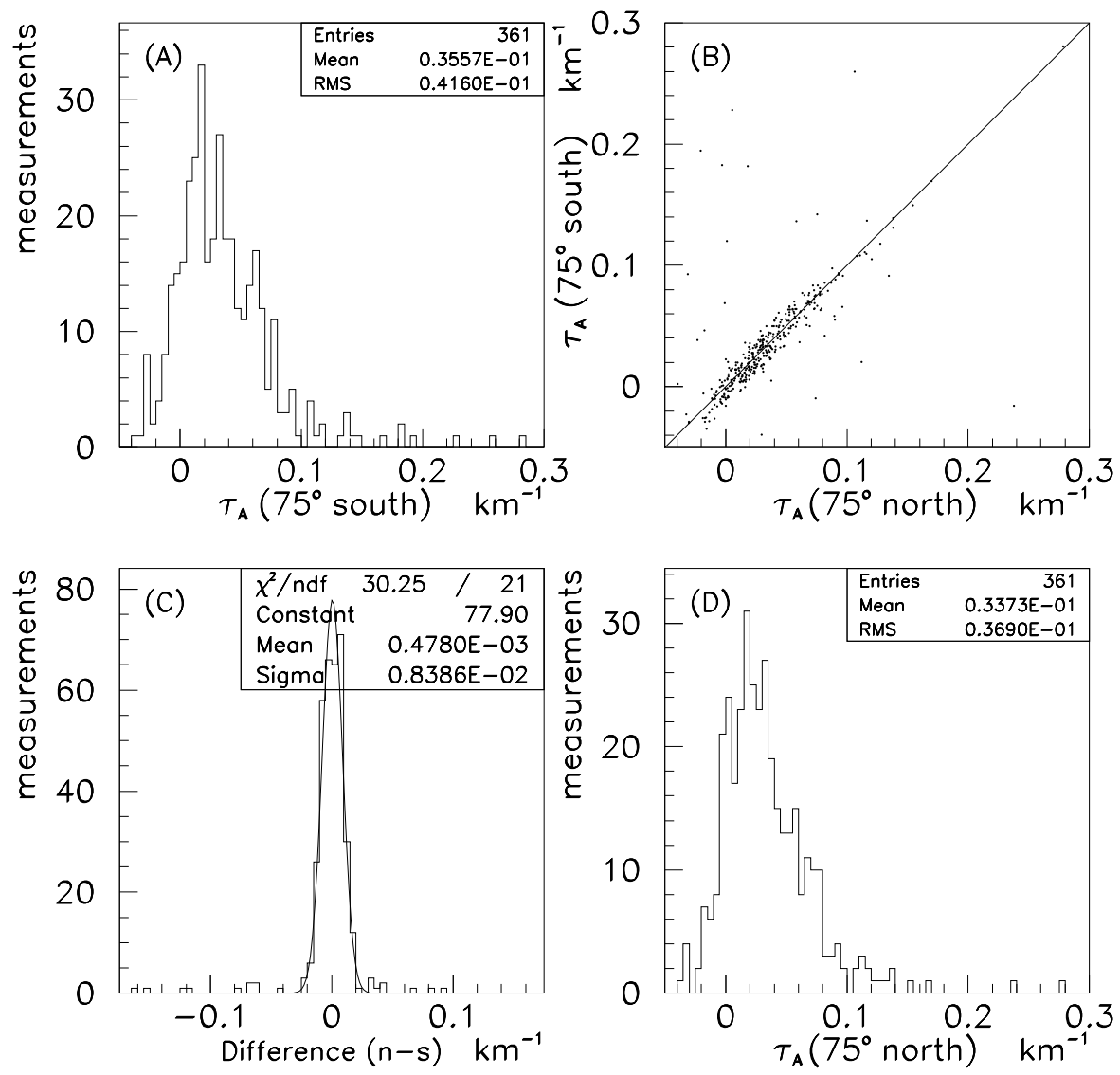

Fig. 5. Comparison of vertical aerosol optical depth from 15-degree inclined laser shots fired at an azimuth angle of 75 degrees to the north (panel A) and 75 degrees to the south (panel D) with respect to HiRes1. The regions of the tracks used for the measurements are separated by roughly $20 \mathrm{~km}$. The correlation (panel B) and difference (panel C) provide a test of aerosol horizontal uniformity.

mirrors of the detector. The left/right differences in $\tau_{A}$ fall within \pm 0.01 (see figure 5) for more than $90 \%$ of the data. A gaussian fit to the difference in these $\tau_{A}$ measurements yields a sigma of 0.008 . In addition, the absolute scale of the average $\tau_{A}$, as measured with inclined tracks, is consistent with the average obtained from the analysis of vertical tracks.

\subsection{Horizontal Attenuation Length and Aerosol Phase Function}

The horizontal attenuation length (HAL) is the aerosol attenuation length at ground level. HAL provides a normalization of the aerosol density. The phase 


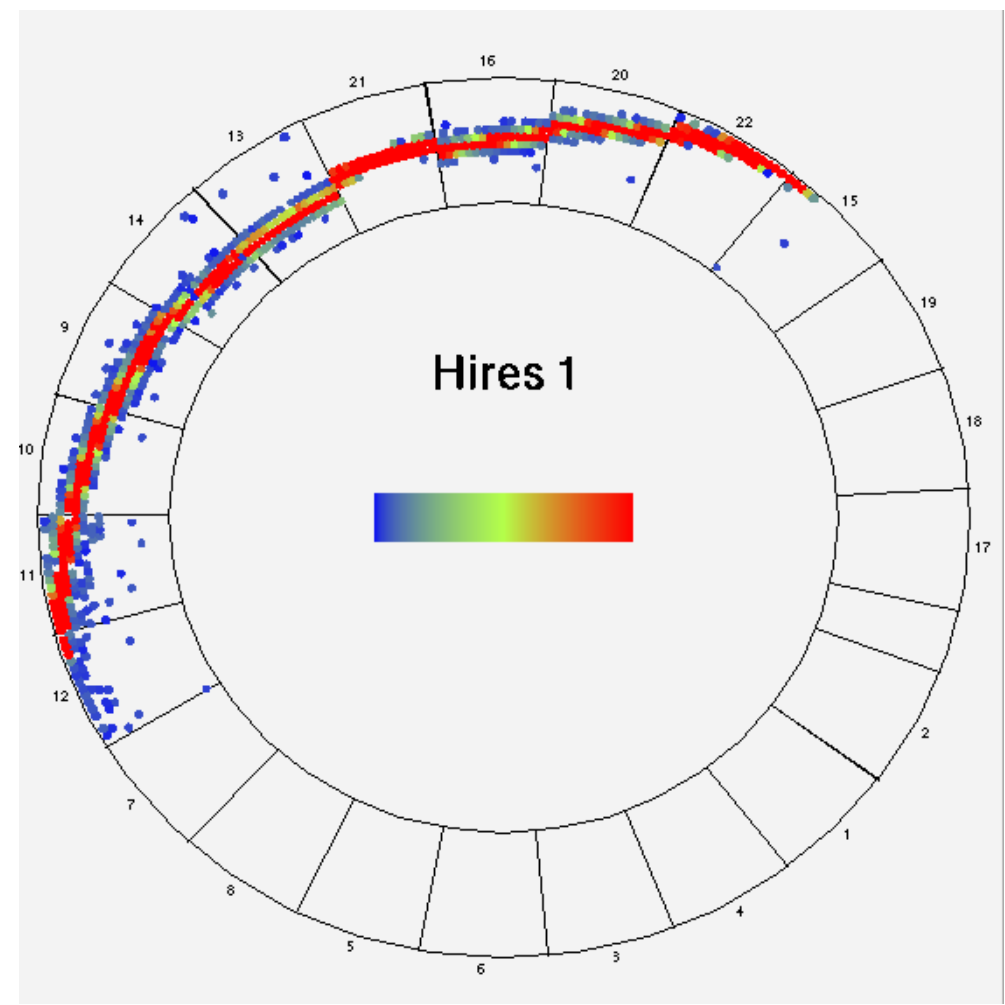

Fig. 6. Track made by nearly horizontal laser shot, as recorded by the HiRes1 detector.

function, or normalized differential scattering cross section, of the aerosols is needed to correct for Cherenkov light scattered from an air-shower. HAL and phase function are measured simultaneously with a fit to a near horizontal shot from the HR2SLS. This shot, passing within $450 \mathrm{~m}$ of the HiRes1 site, generates a long track in the detector (see figure 6) that is observed over a large range of scattering angles. Assuming horizontal uniformity, an aerosol scattering albedo near unity, and known molecular scattering conditions, the longitudinal track profile can be fit to determine HAL and the aerosol phase function (see figure 7).

The functional form of the aerosol phase function is

$$
\frac{d \sigma}{d \Omega} \propto e^{-\mathrm{B} \theta}+\mathrm{C} e^{\mathrm{D} \theta}
$$

where $\theta$ is the scattering angle in radians and $B, C, D$ are free parameters in the fit. This functional form reproduces the shape of a Mie theory-derived phase function except in the very forward scattering region $\left(<2^{\circ}\right)$, where only a small fraction of the total scattered signal appears.

Figure 8 shows the measured distribution of ground-level scattering coefficients 

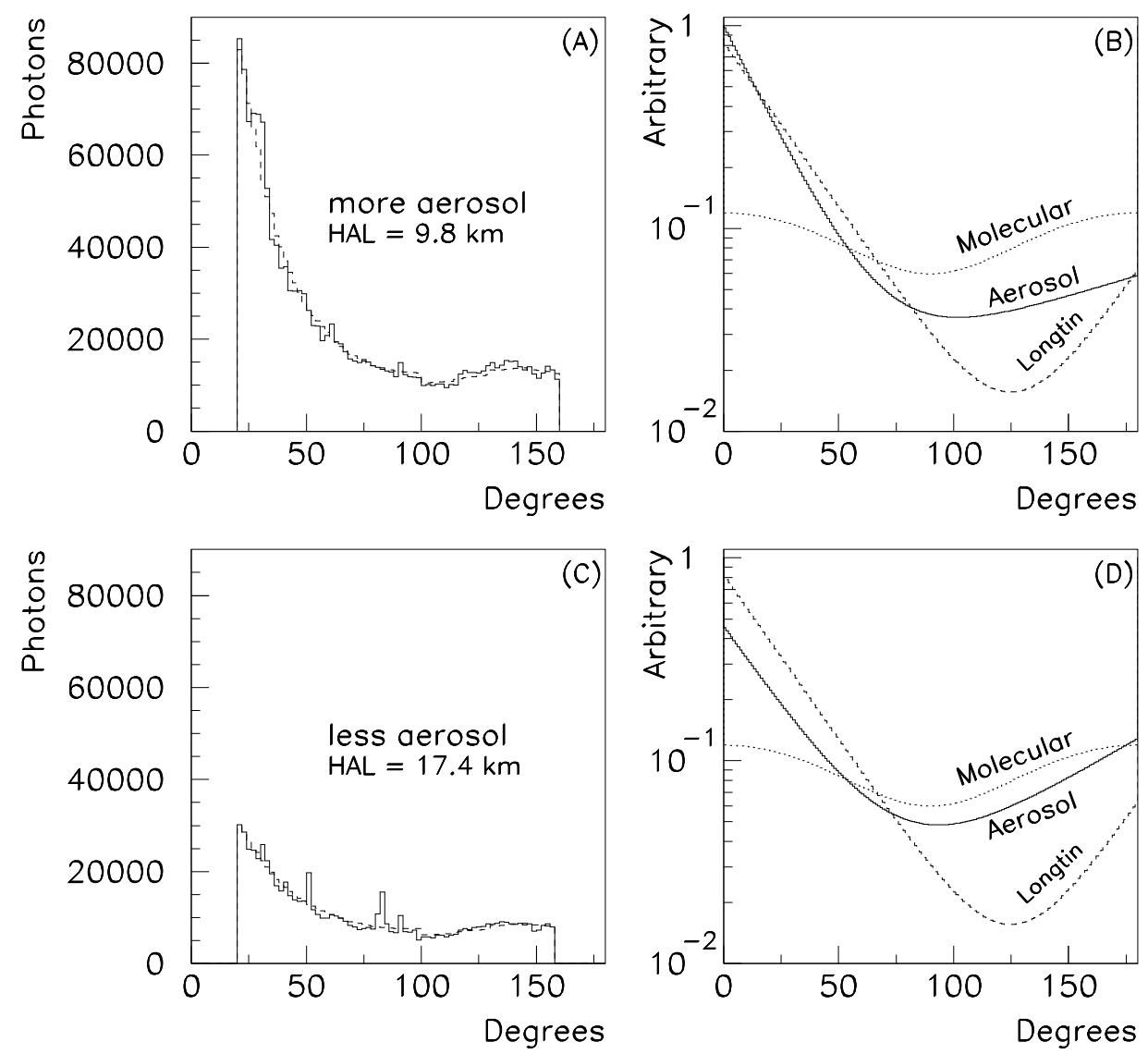

Fig. 7. Two examples of fits for Horizontal Attenuation Length (HAL) and aerosol phase function from data collected under two atmospheric conditions. Panels A and $\mathrm{C}$ show light intensity recorded from near-horizontal laser shots at the HiRes1 detector as a function of scattering angle. Panels B and D show the corresponding aerosol phase function obtained from the fit. For comparison, the molecular phase function and the Mie theory-derived phase function of the Longtin desert aerosol model are also shown.

$\left(\alpha_{0}=\mathrm{HAL}^{-1}\right)$. If the very hazy data $\left(\alpha_{0}>0.1 \mathrm{~km}^{-1}\right)$ are excluded, the average value of $\alpha_{0}$ is $0.04 \mathrm{~km}^{-1}$.

A mean aerosol scale height can be inferred by dividing the mean $\tau_{A}$ by the mean $\alpha_{0}$. This calculation yields $1 \mathrm{~km}$. We note this value is consistent with the differences between the $\tau_{A}$ measurements at different heights as described previously. 

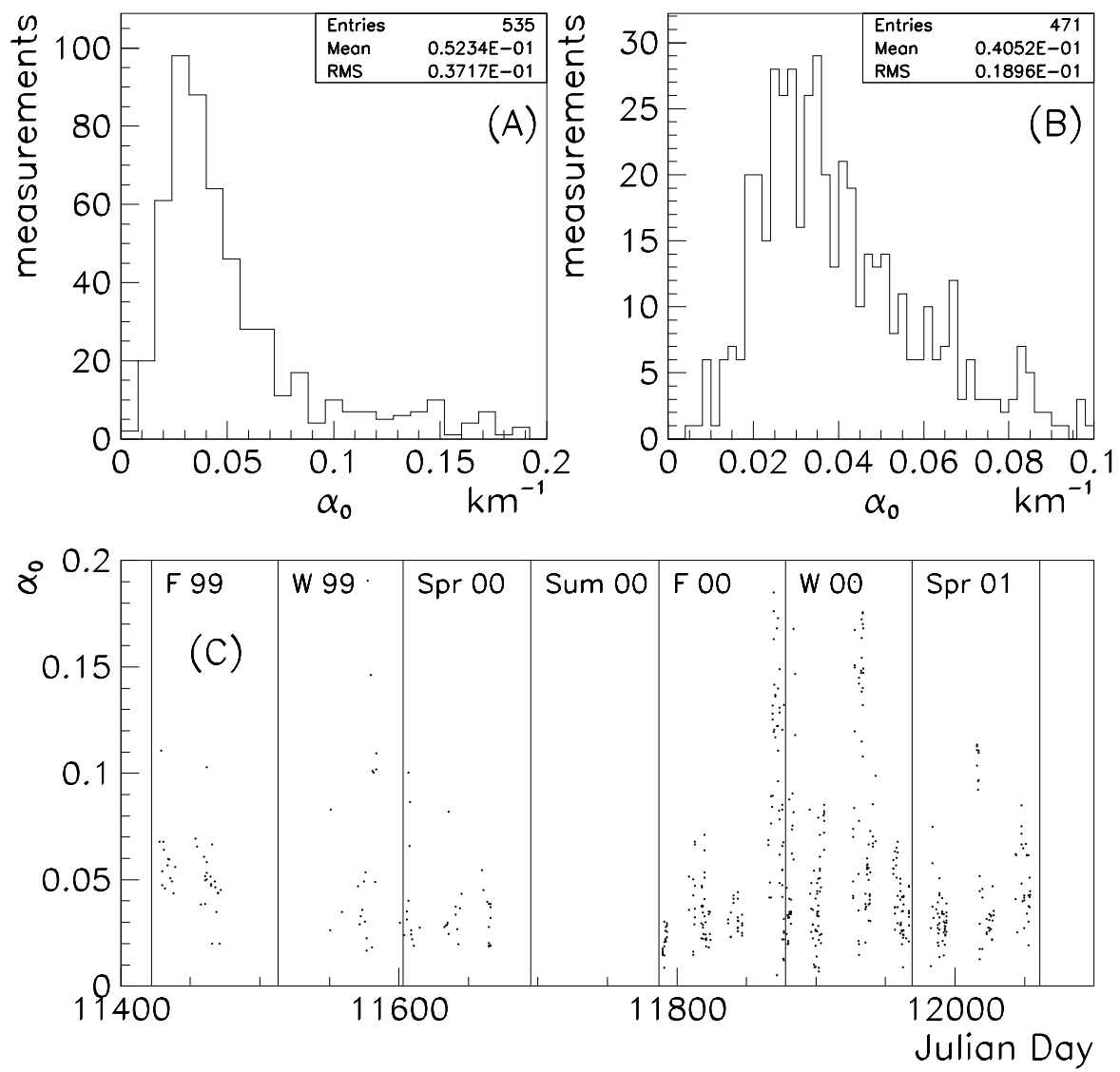

Fig. 8. Measurements of $\alpha_{0}$ from near-horizontal laser shots. $\alpha_{0}$ is the inverse of the horizontal aerosol scattering length at the height of the detector. Panels A and B show the distribution on different scales. The time dependence is shown in panel C.

\section{Checking the Aerosol Optical Depth using Air-Shower Data}

A sample of air-showers observed by both HiRes1 and HiRes2 was used to cross-check the scale of the atmospheric laser measurements. The technique, detailed elsewhere (Abassi et al. (2005)), is based on the principle that multiple eyes viewing a common track segment should reconstruct the same number of emitted photons after corrections for geometrical factors and atmospheric attenuation. If two detectors view the same shower through different optical depths, an error in the atmospheric model can cause an inconsistency between the two detectors in the number of emitted photons from the common track segment. Although this technique is a useful cross-check, the detection rate of air showers is too low to provide precise measurements over a single night. 
For this study, a sample of 415 stereo events recorded between August 25, 2000, and May 23, 2001, was selected. To ensure well-constrained stereo reconstruction, the opening angle between the shower-detector planes was required to be between 8 and 172 degrees. A cut on the minimum viewing angle between the shower axis and the mirror axes of 30 degrees at each detector reduced asymmetric contributions from scattered Cherenkov light. For each event the light-balance $\Delta_{N}$ was calculated from the number of photons reconstructed by the HiRes1 and HiRes2 detectors from the common track segment,

$$
\Delta_{N} \equiv \ln \left(\frac{N_{H i R e s 1}}{N_{H i R e s 2}}\right)
$$

Light-balance is plotted as a function of the linear asymmetry $\Delta_{r}$,

$$
\Delta_{r} \equiv r_{H i R e s 2}-r_{H i R e s 1}
$$

where $r_{\text {HiResX }}$ is the distance between a detector and the center of the common segment.

Figure 9 shows this analysis for three aerosol models: 1) near molecular $\tau_{A}$ $=0.001$; 2$) \tau_{A}=0.040 \mathrm{HAL}=25$; 3) "Standard Desert" $\tau_{A}=0.100 \mathrm{HAL}=$ $12 \mathrm{~km}$. The Standard Desert parametrization leads to an over correction. On average, the far detector reconstructs a "brighter" common segment than does the near detector. Conversely, if one assumes a complete absence of aerosols (i.e. molecular atmosphere), a systematic under correction is observed. The $0.04 \tau_{A} 25 \mathrm{~km}$ HAL model, obtained from laser measurements, falls between these extremes, yielding the smallest slope of the three. A $\tau_{A}$ of 0.055 yields a flat slope. The systematic error in this method is approximately 0.01 .

\section{Conclusions}

We have developed several methods to measure the atmospheric aerosol properties of optical depth, extinction length, and phase function. These methods use side-scattered light from a $355 \mathrm{~nm}$ uv laser that produces tracks in the same optical fluorescence detectors that measure tracks from extensive air showers. We have measured distributions of aerosol optical depth and horizontal extinction length at the location of the High Resolution Fly's Eye experiment. We found the distribution of aerosols is horizontally uniform over a distance of $20 \mathrm{~km}$ to less than 0.01 in $\tau_{A}$ on more than $90 \%$ of the nights observed, and that most of these aerosols are in the lower $2 \mathrm{~km}$ of the atmosphere. Between fall 1999 and spring 2001, the optical depth of the aerosol component is on average less than $25 \%$ of the optical depth of the molecular component. As a 


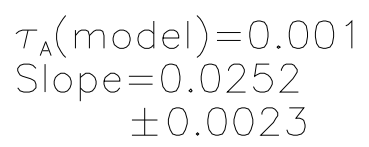

$\tau_{\mathrm{A}}($ model $)=0.040$

Slope $=0.0077$

$\pm 0.0023$

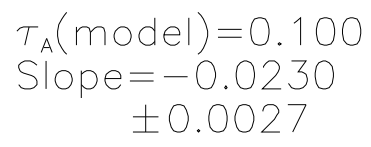

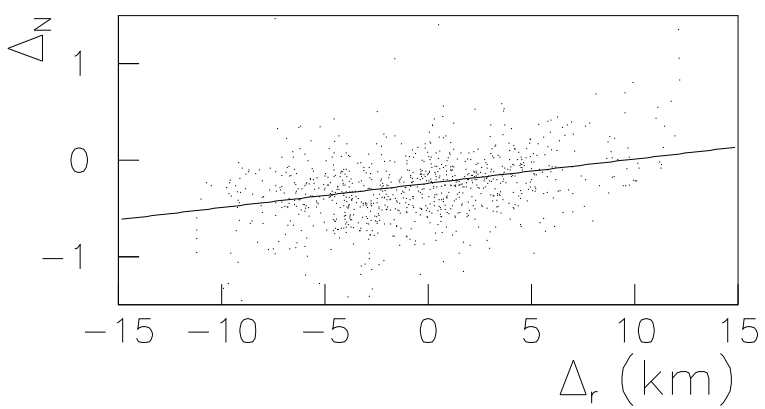
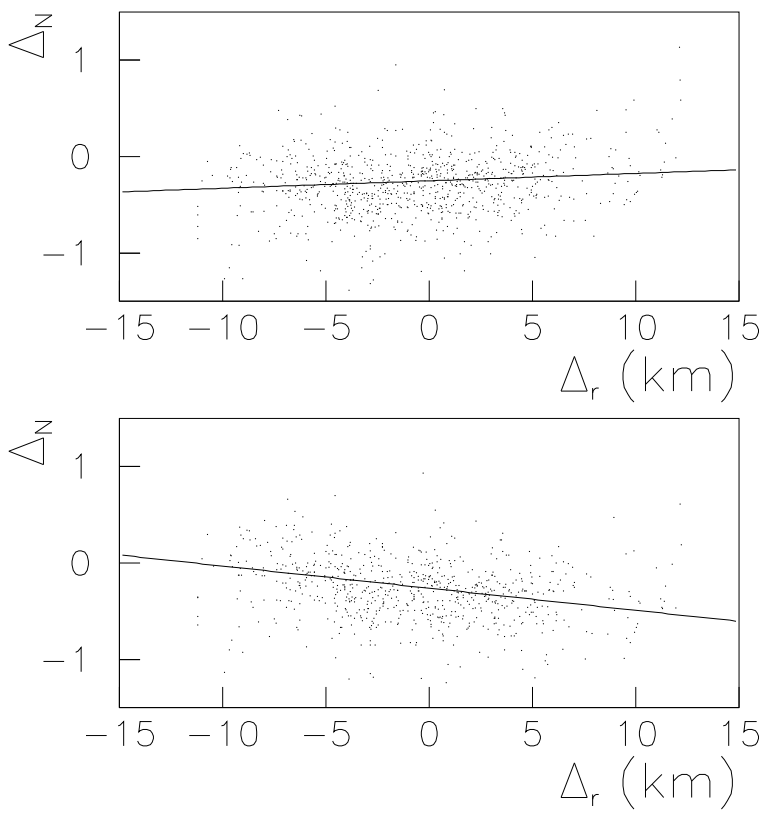

Fig. 9. Light balance as determined by applying three different aerosol models to a single set of stereo events: top panel: near molecular, $\tau_{A}=0.001$; middle panel: $\tau_{A}=$ 0.040 bottom panel: $\tau_{A}=0.100$; (see text).

cross check, we note that the average $\tau_{A}$, as obtained by laser measurements, is consistent an average determined by comparing commonly viewed segments of extensive air showers observed by the two HiRes fluorescence detectors.

\section{Acknowledgments}

This work is supported by US NSF grants PHY-9321949, PHY-9322298, PHY0998826, PHY-024528, PHY-0305516, PHY-0307098, and by the DOE grant FG03-92ER40732. We gratefully acknowledge the contributions of the tech- 
nical staffs at our home institutions. The cooperation of Colonels E. Fischer and G. Hatter, the US Army, and Dugway Proving Ground staff is greatly appreciated.

\section{References}

Abassi et al., in preparation.

T.Abu-Zayyad et al., Nucl. Inst. Meth. A450 (2000) 253.

B. Bodhaine et al., Journal of Atmospheric and Oceanic Technology, 16 (1999) 1854.

C.F. Bohren and D.R. Huffman, "Absoption and Scattering of Light by Small Particles," New York, Wiley Interscience, 1983.

A. Bucholtz, Applied Optics Vol. 34 No. 15 (1995) 2765.

Kakimoto et al., Nucl. Instr. Meth. A372 (1996) 527.

D.R. Longtin, "A Wind Dependent Desert Aerosol Model: Radiative Properties," Air Force Geophysics Laboratory, AFL-TR-88-0112, 1998.

R.B. Miles, W.R. Lempert, and J.N. Forkey, Meas. Sci. Technol. 12 (2001) R33.

Nagano et al., Astroparticle Physics, V22 (2004) 235.

H. Naus and W. Ubachs, Optics Letters Vol. 25 No. 5 (2000) 347.

L. Wiencke, et al, Proc. SPIE Vol 3818, (1999) 46. 